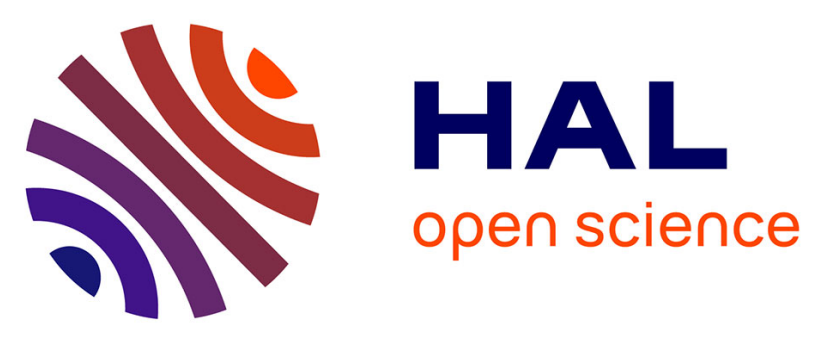

\title{
A hybrid FDTD-Rayleigh integral computational method for the simulation of the ultrasound measurement of proximal femur
}

Didier Cassereau, Pierre Nauleau, Aniss Bendjoudi, Jean-Gabriel Minonzio, Pascal Laugier, Emmanuel Bossy, Quentin Grimal

\section{To cite this version:}

Didier Cassereau, Pierre Nauleau, Aniss Bendjoudi, Jean-Gabriel Minonzio, Pascal Laugier, et al.. A hybrid FDTD-Rayleigh integral computational method for the simulation of the ultrasound measurement of proximal femur. Ultrasonics, 2014, 54 (5), pp.1197-1202. 10.1016/j.ultras.2013.06.004 . hal-01302000

\section{HAL Id: hal-01302000 \\ https: / hal.sorbonne-universite.fr/hal-01302000}

Submitted on 13 Apr 2016

HAL is a multi-disciplinary open access archive for the deposit and dissemination of scientific research documents, whether they are published or not. The documents may come from teaching and research institutions in France or abroad, or from public or private research centers.
L'archive ouverte pluridisciplinaire HAL, est destinée au dépôt et à la diffusion de documents scientifiques de niveau recherche, publiés ou non, émanant des établissements d'enseignement et de recherche français ou étrangers, des laboratoires publics ou privés. 


\title{
A hybrid FDTD-Rayleigh integral computational method for the simulation of the ultrasound measurement of proximal femur
}

\author{
Didier Cassereau ${ }^{\mathrm{a}, \mathrm{b}, *}$, Pierre Nauleau ${ }^{\mathrm{a}}$, Aniss Bendjoudic ${ }^{\mathrm{c}}$.Jean-Gabriel \\ Minonzio $^{\mathrm{a}}$, Pascal Laugier ${ }^{\mathrm{a}}$, Emmanuel Bossy ${ }^{\mathrm{c}}$, Quentin Grimal ${ }^{\mathrm{a}}$ \\ ${ }^{a}$ Laboratoire d'Imagerie Paramétrique, CNRS UMR7623, UPMC Univ Paris6, 15 rue de \\ l'Ecole de Médecine, 75006 Paris, FRANCE \\ ${ }^{b}$ ESPCI ParisTech, 10 rue Vauquelin, 75005 Paris, FRANCE \\ ${ }^{c}$ Institut Langevin, CNRS UMR7587, ESPCI ParisTech, INSERM ERL U979, \\ Université Denis Diderot-Paris7, 1 rue Jussieu, 75005 Paris, FRANCE
}

\begin{abstract}
In the context of development of new quantitative ultrasound (QUS) techniques to probe cortical bone at the hip, interaction of ultrasound with bone need to be modeled. Numerical methods classically used (Finite elements or Finite differences in time domain (FDTD)) are not suited to simulate ultrasonic propagation over long distances. In this paper, an hybrid numerical method is presented to simulate the QUS measurement at the femoral neck. It consists in coupling a FDTD simulation, to compute wave field in the vicinity of bone, with a semi-analytic calculation, to compute wave field between the probe and the bone. This hybrid method is used to simulate a setup dedicated to the measurement of circumferential guided waves in the femoral neck. The proposed approach is validated by comparison with a full FDTD simulation, on the one hand and with a corresponding in vitro experiment on bone phantom, on the other hand. Resulting signals are compared and required computation ressources are estimated, indicating the relevance of this hybrid technique.
\end{abstract}

Keywords: Simulation, FDTD, Semi-analytic, Femoral neck, Guided waves, DORT method

\footnotetext{
${ }^{*}$ Corresponding author

Email address: didier.cassereau@upmc.fr (Didier Cassereau)
} 


\section{Introduction}

Osteoporotic hip fractures are associated with a high mortality and high treatment costs [1]. Fracture risk is best predicted by site-matched measurement of areal bone mineral density (aBMD) with dual-energy X-ray absorptiometry [2]. However, aBMD alone is not sufficient to account for bone strength $[3,4]$. The development of a quantitative ultrasound (QUS) hip scanner $[5,6]$ aims at providing an alternative to aBMD measurements. Because the cortical shell at the proximal femur determines a large part of bone strength $[7,8]$, there is substantial interest in developing QUS measurements with improved sensitivity to cortical bone geometrical and material properties.

The propagation of waves around the circumference of the cortical shell of the femur neck (FN) was first evidenced with numerical simulations $[9,10]$. The interest for these waves was reinforced by the results of a pilot in vitro study [11] which suggested that there is a strong relationship between femur strength and the time-of-flight of the first arriving signal associated with waves traveling circumferentially. Furthermore, it is likely that several modes of circumferential guided waves can be measured in the cortical bone of the FN using multi-emitter and multi-receiver ultrasonic arrays and dedicated signal processing techniques such as the DORT method [12, 13]. This method is suited to measure guided waves dispersion curves of a distant immersed object. These dispersion curves can in principle be processed to derive independent information on bone geometry and material properties.

The development of these novel QUS approaches using guided waves is critically dependent on the possibility to model and simulate the interaction of ultrasound with bone. It is particularly challenging to simulate QUS measurement of the FN because: i) the shape of the $\mathrm{FN}$ is complex and ii) the ultrasound probe is necessarily placed at a large distance from the bone, that is, the ultrasound beam must travel through a large thickness of soft tissues. In order to model the shape of the FN, numerical methods based on a discretization of space like finite elements or finite difference methods must be used. However these methods are in general not adapted to simulate the propagation over large distances (i.e. large number of wavelenghts) in homogeneous or quasi-homogeneous media (soft tissues) because of the accumulation of numerical errors along the wave path. Also, in many cases it 
is important to keep the computation time as low as possible because of the necessity to repeat the simulations for a given configuration to account for multiple emissions when multi-emitter arrays are used.

The aim of this paper is to present the implementation of an hybrid numerical method to simulate the QUS measurements at the FN. The method consists in coupling a finite difference time domain (FDTD) simulation to compute the ultrasound wave field in the vicinity of the FN and a semianalytic technique which evaluates the Rayleigh integral to compute the wave propagation between the ultrasonic array and the FDTD domain. In this paper we consider the simulation of a set-up which is dedicated to the measurement of circumferential guided waves, but the proposed hybrid method is not restricted to this configuration.

\section{Configuration and model}

We consider the modeling of a measurement set-up which aims at recording the wave field scattered by the cortical bone shell of the FN (fig.1).[13] The transducer is a phased array of 128 independent elements working in the transmit/receive mode. It is cylindrically pre-focused in order to concentrate the beam energy in a plane perpendicular to the FN axis, which is placed at the focal distance. A similar setup with a monoelement probe was used previously to measure femurs in vitro [11].

The bone is supposed to be immersed in a homogeneous fluid with the properties of water which represents soft tissues and coupling medium between the array and the skin. Cortical bone is modeled as an elastic solid. Trabecular bone and marrow in the cortical shell are not modeled for the sake of simplicity, i.e. the interior of the shell is modeled as void.

For the purpose of simulating the measurement of the ultrasound field scattered by the bone shell, the physical space is split in two domains: $\mathcal{A}$ is a rectangular area in the vicinity of the bone; $\mathcal{B}$ designates the remaining of space and in particular the space between the transducer and domain $\mathcal{A}$.

The model is three-dimensional in $\mathcal{B}$ and two-dimensional in $\mathcal{A}$. The two-dimensional approximation consists in neglecting the diffraction of the incident beam in the $\mathbf{z}$-direction, which is motivated by the fact that the probe is pre-focused in a plane perpendicular to the FN axis and that the geometry of the FN is close to that of a cylinder. 


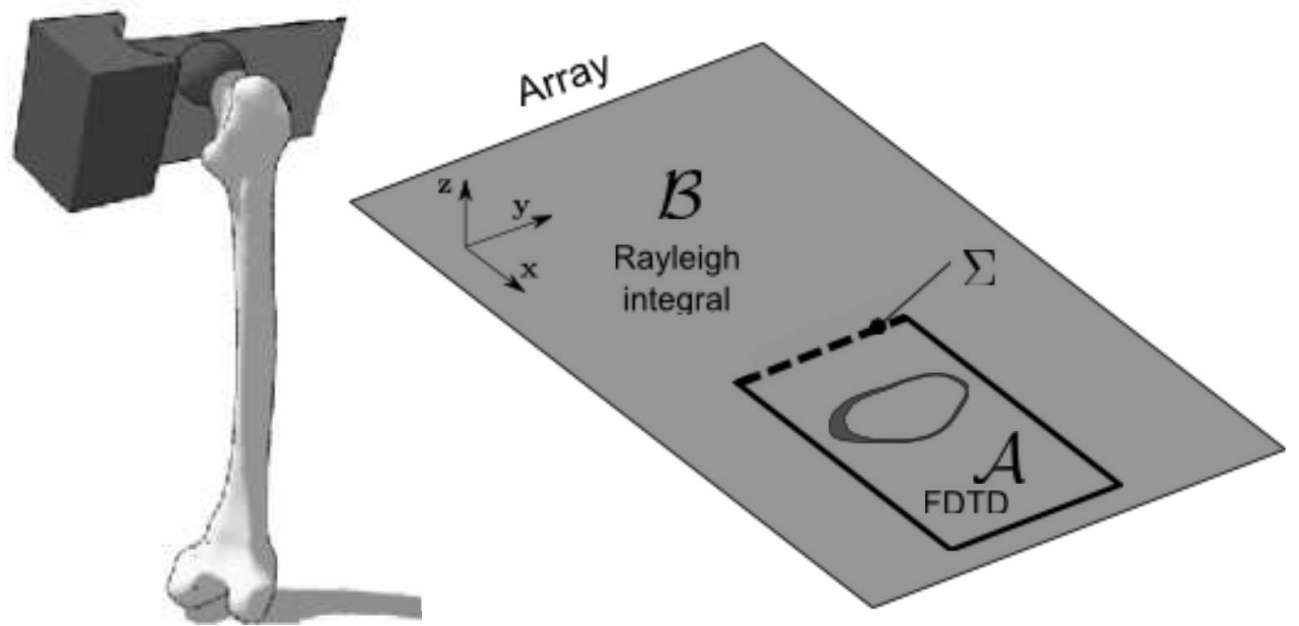

Figure 1: Representation of the configuration for the measurement of the wave field backscattered by the femoral neck cortical shell (left). The measurement is performed in the focal plane of the pre-focused array which intersects a cross-section of the neck (gray area). $\mathcal{A}$ designates an area in the vicinity of the bone cross-section where the wave field is computed with FDTD; $\mathcal{B}$ designates a homogeneous fluid domain in which the ultrasound propagation between the array and the coupling line $\Sigma$ is calculated by evaluating the Rayleigh integral (right).

\section{Hybrid FDTD/Rayleigh integral computational method}

\subsection{FDTD}

In domain $\mathcal{A}$ the elastodynamics equations

$$
\begin{array}{r}
\rho(\mathbf{x}) \frac{\partial v_{i}}{\partial t}(\mathbf{x}, t)=\sum_{j=1}^{d} \frac{\partial \sigma_{i j}}{\partial x_{j}}(\mathbf{x}, t) \\
\frac{\partial \sigma_{i j}}{\partial t}(\mathbf{x}, t)=\sum_{j=1}^{d} \sum_{i=1}^{d} c_{i j k l}(\mathbf{x}) \frac{\partial v_{k}}{\partial x_{l}}(\mathbf{x}, t)
\end{array}
$$

are discretized and the wave field is computed with the FDTD method implemented in Simsonic software [14]. This sofware implements Virieux's scheme $[15,16]$. In Eqs. (1-2), $\rho$ is the density, $v_{i}$ are the components of the particle velocity, $c_{i j k l}$ are the components of the stiffness tensor, and $\sigma_{i j}$ are the components of the stress tensor. These quantities are defined at each point $\mathbf{x}$ of the FDTD grid. The spatial domain is discretized with an isotropic grid step 
$\Delta x$. The temporal step $\Delta t$ for time discretization must satisfy the stability condition (usually known as the Courant, Friedrichs and Levy condition)

$$
\Delta t \leq \frac{1}{\sqrt{2}} \times \frac{\Delta x}{c_{\max }}
$$

where $c_{\max }$ is the largest propagation velocity in domain $\mathcal{A}$. The accuracy of the FDTD numerical computation depends on the choice of $\Delta x$. This choice results of a compromise between accuracy, computation time, and available resources.

\subsection{Rayleigh integral}

The propagation of waves in domain $\mathcal{B}$, in particular between the transducer and domain $\mathcal{A}$, can be treated in the framework of the standard impulse diffraction formulation in an homogeneous fluid. In the case of a planar vibrating surface mounted in an infinitely rigid baffle, the transient diffracted beam can be calculated using Green's functions approach $[17,18]$. The velocity potential at point $\mathbf{r}$ due to each element of the array writes

$$
\Phi(\mathbf{r}, t)=\varphi(t) \frac{*}{t} h(\mathbf{r}, t),
$$

where $* / t$ denotes the temporal convolution, $\varphi(t)$ is the excitation function and $h(\mathbf{r}, t)$ is the impulse Rayleigh integral defined by

$$
h(\mathbf{r}, t)=\frac{1}{2 \pi} \int_{S} V_{n}\left(\mathbf{r}_{s}\right) \frac{1}{\left|\mathbf{r}-\mathbf{r}_{s}\right|} \delta\left(t-\frac{\left|\mathbf{r}-\mathbf{r}_{s}\right|}{c}\right) d^{2} \mathbf{r}_{s}
$$

In Eq. (3), $\mathbf{r}_{s}$ denotes the integration point over the active aperture $S$ of the element, $V_{n}\left(\mathbf{r}_{s}\right)$ is the normal velocity distribution on the surface of the element supposed to act as a piston, $c$ is the speed of sound in the fluid, and $\delta(t)$ is the Dirac delta function. The acoustic pressure results from the standard relationship $p(\mathbf{r}, t)=-\rho_{f} \frac{\partial \Phi(\mathbf{r}, t)}{\partial t}$, where $\rho_{f}$ is the density of the fluid.

An additional approximation is sometimes possible, that consists in considering that $V_{n}\left(\mathbf{r}_{s}\right)$ does not depend on the position $\mathbf{r}_{s}$ on the vibrating surface, such that the normal displacement on the surface of the transducer is uniform. In this case, a closed-form expression of the impulse diffraction response $h(\mathbf{r}, t)$ can be obtained for particular geometries of the radiating surface, like a disk or a rectangle $[18,19]$. In practice, we do not consider this particular case. 
The computation of the diffracted beam in the far field-at least at the spatial scale of each individual element of the phased array-in the domain $\mathcal{B}$ is performed with PASS (Phased Array Simulation Software [20]) that implements a discretization of the impulse Rayleigh integral (Eq. (3)). The spatial grid size for this discretization depends on the distance between the observation point and the radiating surface and on the central frequency and bandwidth of the excitation function $\varphi(t)$. With this method, it is sufficient to compute the beam at the selected points of interest, and not the complete wave field on the whole space domain.

It is important to note that the Rayleigh integral is strictly valid only in the case of planar sources, while the elements of the considered array have a cylindrical curvature. However, it can be shown that the Rayleigh integral remains valid for a small aperture in the far-field domain [21], and this condition is effectively satisfied in our experimental setup.

\subsection{Coupling of FDTD and Rayleigh integral calculations}

The integral formulation of acoustics boundary value problems provides the theoretical framework to perform the coupling between wave fields in domains $\mathcal{A}$ and $\mathcal{B}$ [22, p. 320][23]. On the one hand, it can be shown that the wave field in $\mathcal{A}$ can be calculated based on the sole knowledge of the acoustic pressure on the domain boundary. This pressure is calculated by PASS. On the other hand, it can be shown that the backscattered wave field received by the transducer can be calculated based on the sole knowledge of the acoustic velocity normal to the boundary of a closed domain, within $\mathcal{B}$, enclosing the array (equivalent to using Eq. (3)).

In practice, two assumptions are made: (A1) the coupling between wave fields in $\mathcal{A}$ and $\mathcal{B}$ is only effective through the 'coupling' interface $\Sigma$ (Figs. 1), i.e. it is assumed that the waves radiated through the rest of the boundaries of $\mathcal{A}$ and $\mathcal{B}$ can be disregarded; (A2) there is no time overlap between emitted waves traveling in the direction $\mathcal{B} \rightarrow \mathcal{A}$ and backscattered waves traveling in the direction $\mathcal{A} \rightarrow \mathcal{B}$.

The general computational workflow is: (1) calculation of the Rayleigh integral with PASS to evaluate acoustic pressure on $\Sigma$ due to transient signals emitted by the array; (2) pressure is used as a forced boundary condition in the FDTD code. During phase (2), the forced pressure condition is released as soon as the incident wave has traveled through the interface, before any backscattered wave reaches the interface (see assumption A2); (3) the normal velocity on $\Sigma$ due to the backscattered wave field is calculated with FDTD 


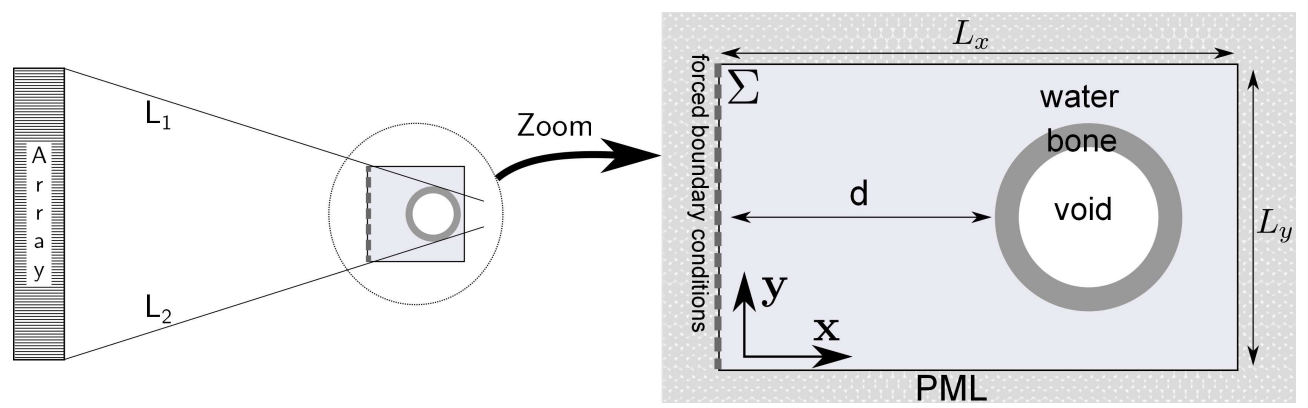

Figure 2: Left: Configuration of a DORT in vitro experiment on a tube made of bone mimicking material. Lines $\mathrm{L}_{1}$ and $\mathrm{L}_{2}$ are attached to the first and last elements of the array and tangent to the shell. They define the length, $L_{y}$, of the coupling line. Right: Zoom on domain $\mathcal{A}$. Configuration for the FDTD computation of the interaction of ultrasound with the studied phantom.

in $\mathcal{B}$. The backscattered waves encounter perfectly matched layers (PML) which prevent any reflection of the waves on the box boundaries [24]; (4) this velocity is then used in PASS to calculate the wave field back-propagated to the receiver.

The implementation of the coupling of FDTD and Rayleigh integral calculation described above must be adapted for a given configuration in order to comply with assumptions (A1) and (A2). In particular, the choice of the size of $\mathcal{A}$ is critical: the smaller the domain $\mathcal{A}$, the smaller the computation time. Note that choosing the length and position of the coupling line $\Sigma$ with respect to the bone shell essentially determines the dimensions of the FDTD box.

In the present work, the hybrid method was applied to the measurement of the signals backscattered by a cylindrical shell of circular cross-section (Fig. 2). The method to choose the length along axis $\mathbf{y}$ of the line $\Sigma$ and the distance $d$ between the latter and the shell considers the following: (1) Distance $d$ is such that the duration of the signal emitted by the ensemble of the elements of the array on $\Sigma$ is shorter than the time for a round-trip of acoustic waves between $\Sigma$ and the shell. This ensures that emitted and backscattered signals do not overlap on $\Sigma$. (2) At distance $d$ of the shell, $\Sigma$ is bounded by two lines attached to the first and last elements ( $L_{1}$ and $L_{2}$ Fig. 2) of the array and tangent to the shell. This ensures that the contributions of all acoustic rays which can be drawn between the array and the shell are taken into account by the coupling, i.e. are actually transmitted in $\mathcal{A}$. 


\section{Simulations}

\subsection{Parameters of the simulations}

The shell is a circular tube (Fig. 2) of external diameter $26 \mathrm{~mm}$ and thickness $2.1 \mathrm{~mm}$ made of a bone mimicking material with longitudinal velocity $c_{l}=2870 \mathrm{~ms}^{-1}$, transverse velocity $c_{t}=1520 \mathrm{~ms}^{-1}$ and density $\rho_{s}=1.64 \mathrm{gcm}^{-3}[25]$. Properties of water are: density $\rho=1$ and velocity $c=1500 \mathrm{~m} / \mathrm{s}$. The excitation function is a pulse of $1 \mathrm{MHz}$ central frequency with $-6 \mathrm{~dB}$ bandwidth of $100 \%$.

Two sets of simulations were performed. (1) The validation of the hybrid method and the analysis of the influence of the spatial grid size $\Delta x$ on the numerical results and computation times is investigated with a 'small' computational domain (section 4.2) in order to save computational resources. That is, the modeled distance between the probe and the shell and probe aperture are smaller than in the real experiment. [13] (2) The comparison of the simulation results with experiments on a realistic computational domain is presented in section 4.3 .

\subsection{Validation of the hybrid method}

The tube was located at a distance of $47 \mathrm{~mm}$ from the surface of the probe, which was a 128-element array of aperture $50 \mathrm{~mm}$, prefocused cylindrically in plane $\mathbf{x}-\mathbf{y}$ with a focal length of $60 \mathrm{~mm}$. We considered a spatial domain of width $50 \mathrm{~mm}$ and height $75 \mathrm{~mm}$. The dimensions of the FDTD domain with the hybrid method are $L_{x}=44 \mathrm{~mm}, L_{y}=39 \mathrm{~mm}$. The position of the shell is fixed at $d=17 \mathrm{~mm}$ (Fig. 2).

Simulations were performed with the hybrid method and a 'full' FDTD approach in which the propagation in the entire physical domain between the probe and the cylinder was computed with FDTD. Simulations were run successively for three values of FDTD grid sizes: $\Delta x=50,25$, and $12.5 \mu \mathrm{m}$. Compared with the wavelength in water calculated at the central frequency of the probe $\left(\lambda_{c}=1.5 \mathrm{~mm}\right)$, the three grid step sizes are equal to $\lambda_{c} / 30, \lambda_{c} / 60$ and $\lambda_{c} / 120$, respectively.

We computed the backscattered response when the tube is illuminated by a plane wave (all emitters in phase). This essentially consists of a specular echo and of the contributions of circumferential elastic waves radiating from the surface of the tube.

Fig. 3 shows the portion of the signals calculated on the central element of the array in the time-window in which the contribution of circumferential 


\begin{tabular}{|l|c|c|c|}
\hline & $\Delta x=50 \mu \mathrm{m}$ & $\Delta x=25 \mu \mathrm{m}$ & $\Delta x=12.5 \mu \mathrm{m}$ \\
\hline Hybrid model & $6 \mathrm{~min}$. & $28 \mathrm{~min}$. & $141 \mathrm{~min}$. \\
Full FDTD & $14 \mathrm{~min}$. & $84 \mathrm{~min}$. & $510 \mathrm{~min}$. \\
Ratio & 2.3 & 3 & 3.6 \\
\hline
\end{tabular}

Table 1: Computation times in minutes for the hybrid and the full FDTD approaches for three spatial grid step sizes $\Delta x$. The distance between the probe and the scattering object was $47 \mathrm{~mm}$.

waves is observed. The discrepancies between the signals computed for the three values of $\Delta x$ can be due to the effects of numerical dispersion, as well as the effects of an inacurate description of the curved geometry of the tube in a Cartesian coordinate system ('staircase effect'). Decreasing $\Delta x$ should reduce both these effects. Although it is not possible to evaluate their respective contribution in the present study, we expect that effects of numerical dispersion are smaller than the effects of the curved geometry. Indeed, we found previously that for $\Delta x=\lambda_{c} / 60$ the numerical dispersion is negligible for a propagation in bone and water along a wave path of a few centimeters [26].

The signals resulting from the hybrid and the full FDTD approaches were almost superimposed (Fig. 3), which validates the principles and the implementation of the hybrid scheme. The computation times on an 4-core dual processor computer (64-bit Xeon, $3 \mathrm{GHz}$ ) are summarized in Tab. 1.

\subsection{Measurement of dispersion curves of circumferential waves}

The tube was located at a distance of $160 \mathrm{~mm}$ from the surface of the probe, which was a 128-element array (element size is $1.1 \mathrm{~mm}$ ) of aperture $140 \mathrm{~mm}$, prefocused cylindrically in plane $\mathbf{x}-\mathbf{y}$ with a focal length of $160 \mathrm{~mm}$. The dimensions of the FDTD domain are $L_{x}=47 \mathrm{~mm}, L_{y}=46 \mathrm{~mm}$, and $d=20 \mathrm{~mm}$ (Fig. 2). This corresponds to the experimental conditions used to measure bone phantoms in vitro [13]. In the present section, the results of the hybrid simulation are compared with the experimental results of this latter study.

The comparison of experimental and simulated signals when the tube is illuminated by a plane wave (all emitters in phase) is shown Fig. 4. Computation for one of the 128 emissions typically lasts from 2 to 3 hours, depending on calculation parameters. 


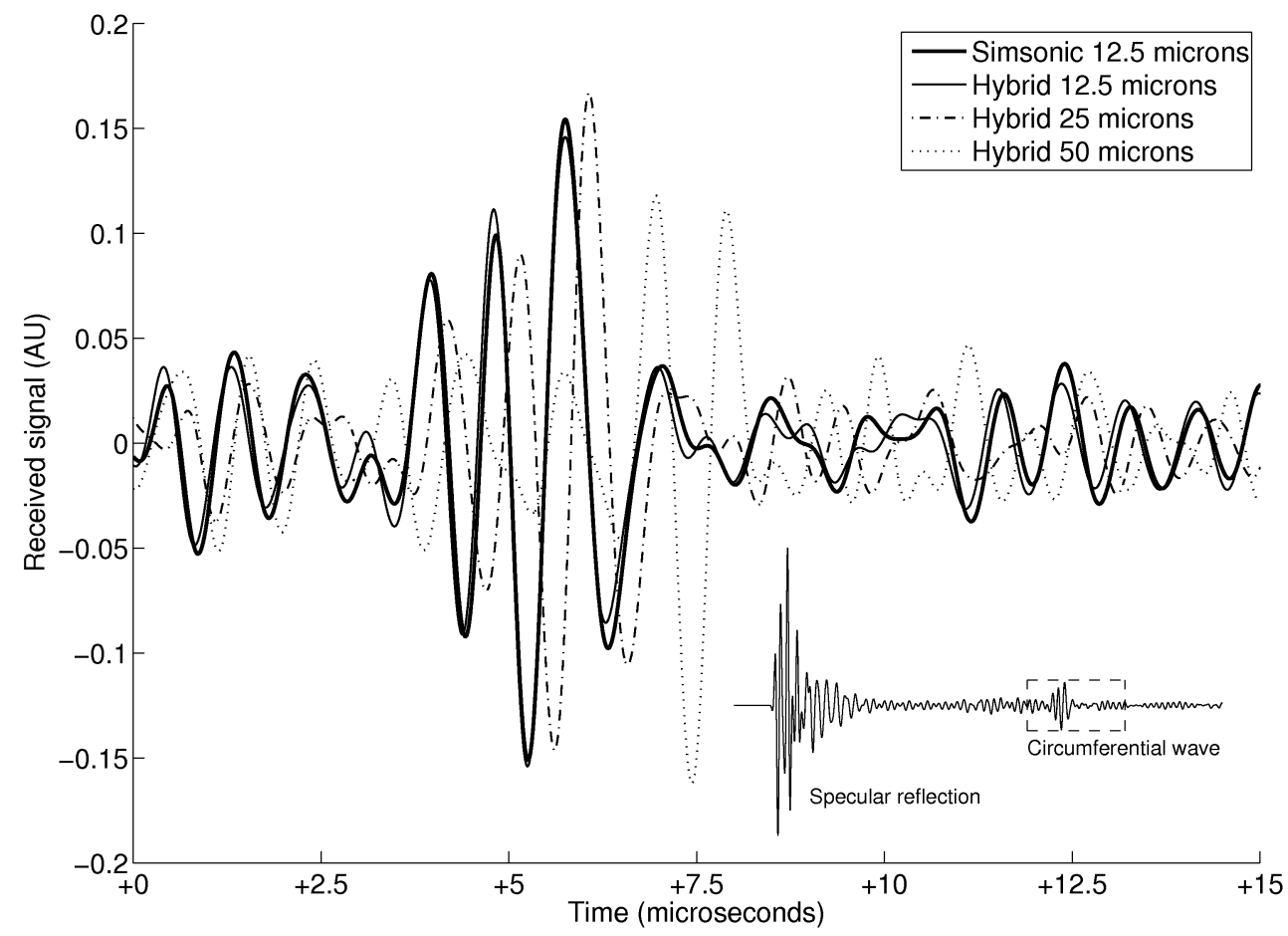

Figure 3: Portion of the backscattered signal calculated on the central element of the array showing the contribution of the circumferential wave. Time is given relative to an arbitrary origin. The dotted, dashed and thin continuous lines correspond to hybrid computations with $\Delta x=50,25,12.5 \mu \mathrm{m}$, respectively. The thick continuous line corresponds to the full FDTD computation with $\Delta x=12.5 \mu \mathrm{m}$. The portion of signal was extracted from the complete signal comprising the specular reflection and the circumferencial wave arriving at a somewhat later time (insert).

The first step of the method to measure the guided waves dispersion curves consists in recording the inter-element impulse response functions and store them in a matrix which is post-processed with the DORT method.[27] This allows to isolate the contribution of the circumferential guided waves in the scattered wave field. The principle of the method, which was described elsewhere $[28,13]$, is as follows. The time-reversal operator is constructed with the Fourier transform of the impulse response matrix. The eigenvectors of this operator are backpropagated numerically, which allows to determine the location of the effective radiation points of each circumferential guided wave mode at each frequency. Under the hypothesis of geometrical acoustics, the location of these points can be related by an explicit equation to a value 


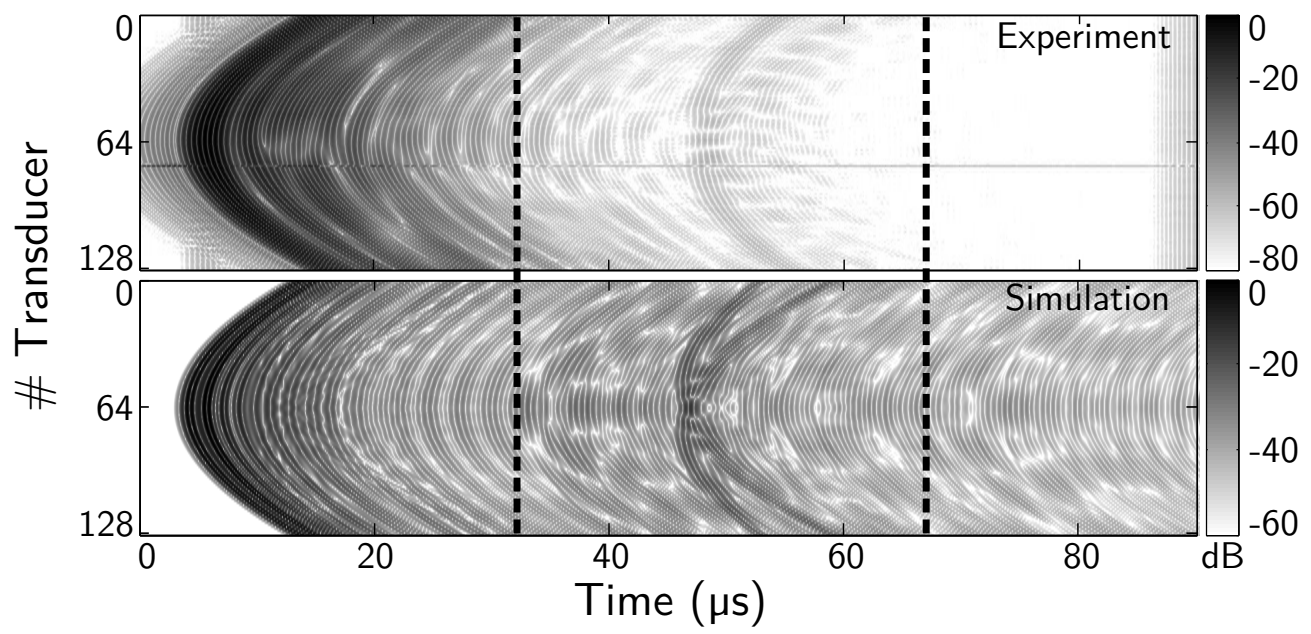

Figure 4: Comparison of experimental and simulated signals (time origin is arbitrary). The tube was illuminated by a plane wave. The specular reflection starts around $5 \mu s$ and circumferential waves around $50 \mu \mathrm{s}$. The time-window between the discontinuous lines was processed to derive dispersion curves. The non-zero signal ahead of the specular wave front in the experimental signals is a signal processing artefact which does not influence the measurement of guided waves.

of the phase velocity $c_{\phi}$. The portions of dispersion curves of circumferential guided waves in the tube obtained with hybrid simulation and in experiments reported in [13] are consistent (Fig. 5).

\subsection{Discussion and Conclusion}

An implementation of an hybrid numerical method for the simulation of a backscattering experiment was presented. The measurement configuration considered is a model of the measurement of the upper extremity of the femur. In the QUS application, the probe is typically at a distance of more than $15 \mathrm{~cm}$ from the femur [5]. In first approach soft tissues and coupling medium between the probe and femur may be approximated by a homogeneous medium, e.g. water. It follows that the simulation of the experiment requires to calculate the propagation of waves in a homogeneous medium over a very large number of wavelengths. A code implementing a semi-analytical evaluation of the Rayleigh integral (software PASS) was used to calculate the wave field in the homogeneous medium (water) at selected points only-and not in the whole domain-which is a key to reduce significantly the computer resources required for the simulation. In the vicinity of 


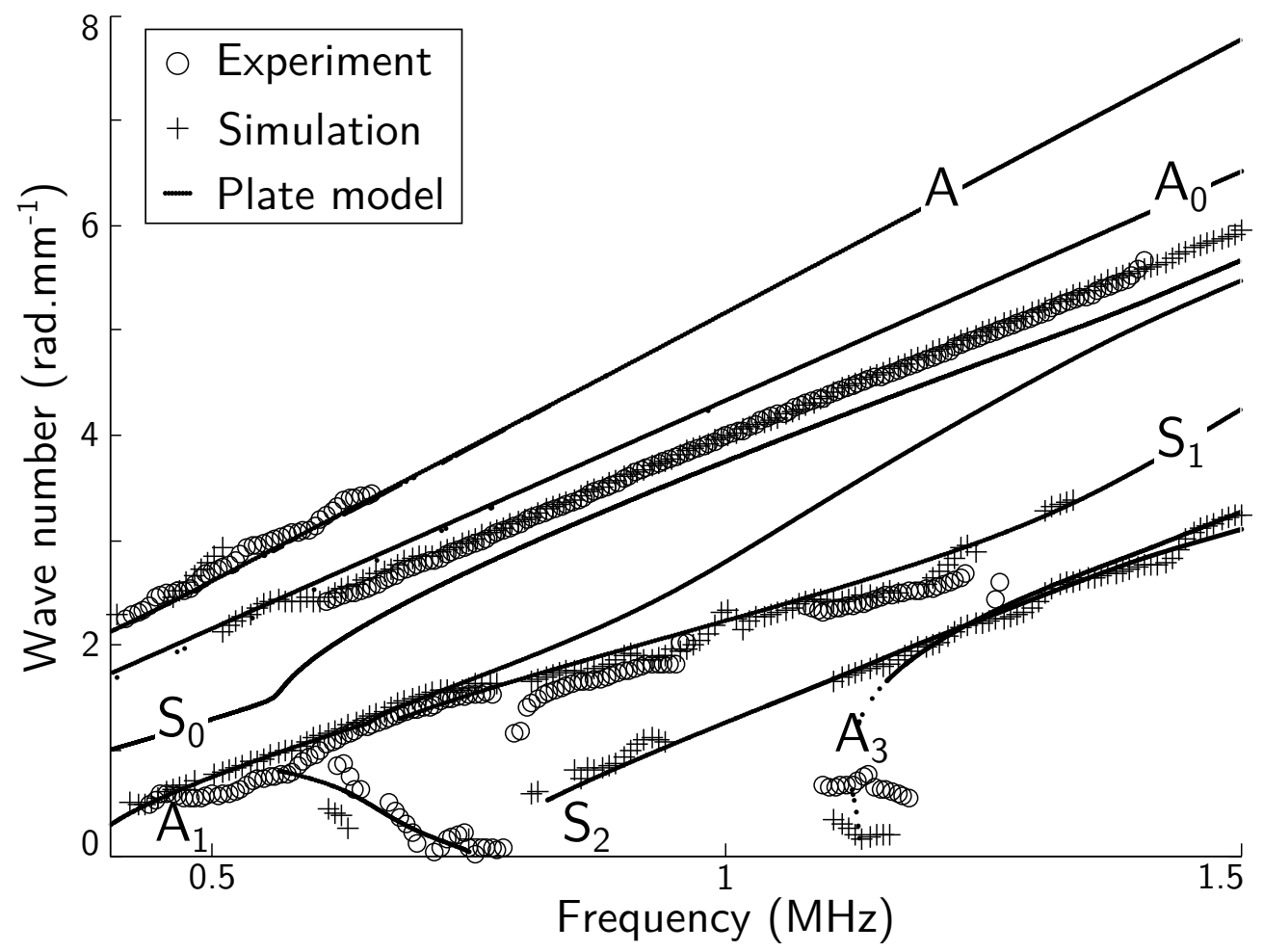

Figure 5: Dispersion curves of circumferential guided waves in the tube obtained with hybrid simulation and in experiments reported in [13]. For reference, dispersion curves for a free plate loaded by water on one side are given. $A_{i}$ and $S_{i}$ respectively denote antisymmetric and symmetrical Lamb modes. See [13] for details.

the scattering object (bone or bone phantom) the wave field is calculated at every point of a discretized domain by FDTD (software Simsonic). With the spatial grid size of $\Delta x=12.5 \mu \mathrm{m}$, for which we obtained the most reliable results, the computation time of the full FDTD computation on a 'small' domain (the modeled distance between the probe and the scattering object was only $47 \mathrm{~mm}$, section 4.2) was 510 minutes. Based on this value, the computation time required for a full FDTD computation corresponding to an actual experimental configuration (the distance between the probe and the scattering object was $160 \mathrm{~mm}$, section 4.3) can be estimated to more than 120 hours. This duration is about sixty times the duration of our proposed hybrid computation. It follows that it will in general be necessary to resort to an hybrid method to simulate a femur QUS measurement. The computation time given above are for a single emission of the transducer. It is all the more 
important to resort to an hybrid method to simulate measurements with the DORT method which involves the computation of the backscattered signal for several different emissions (128 in the present study).

Some of the limitations of the hybrid method presented in the paper follow from the hypotheses underlying the calculation of the Rayleigh integral and Eq. (2) on which the FDTD scheme is based. In particular attenuation in bone and water was not modeled. The importance of attenuation in a bone phantom (Sawbones, Pacific Research Laboratory Inc., Vashon, WA) can be noticed in Fig. 4 where the loss of amplitude in experimental signals is manifest. With the regular grid of the FDTD scheme, the modeling of a curved geometry like a bone cross-sections is cumbersome. The grid step $\Delta x$ should be sufficiently small to model the geometry accurately. Note that alternative FDTD schemes which have been proposed to model explicitly curved boundaries $[29,30]$ could be used advantageously to model QUS experiments. Finally, the method of coupling between the Rayleigh integral formulation and the FDTD assumes that the diffracting object inside the FDTD box is in the far field. Inded the reasoning for the choice of the dimensions of the FDTD box uses the notion of acoustic rays traveling in straight line. The far field assumption is justified in the case studied where the illuminated tube is located at more than 100 wavelengths of the probe.

An increasing number of studies describing the numerical simulation of QUS experiments have been published in the past decade and several groups worldwide have developed dedicated FDTD codes [31, 32, 33, 34]. In this paper we have shown that the simulation of the measurement at the femoral neck is not practicable with FDTD alone. As an alternative we have proposed an hybrid method. The method was validated in a typical QUS experimental configuration and the required computer resources were estimated. In an in vivo measurement of the proximal femur, the ultrasound beam may be diffracted and attenuated by the layers of fat and soft tissues. The proposed hybrid method should be adapted to account for these phenomena.

\section{References}

[1] S. R. Cummings, L. J. Melton, Epidemiology and outcomes of osteoporotic fractures, Lancet 359 (2002) 1761-7.

[2] D. Marshall, O. Johnell, H. Wedel, Meta-analysis of how well measures of bone mineral density predict occurrence of osteoporotic fractures, Brit. 
Med. J. 312 (1996) 1254-9. 0959-8138 (Print) Journal Article MetaAnalysis Research Support, Non-U.S. Gov't.

[3] K. L. Stone, D. G. Seeley, L.-Y. Lui, J. A. Cauley, K. Ensrud, W. S. Browner, M. C. Nevitt, S. R. Cummings, O. F. R. Group, BMD at multiple sites and risk of fracture of multiple types: long-term results from the Study of Osteoporotic Fractures, J. Bone Miner. Res. 18 (2003) 1947-1954.

[4] E. S. Siris, Y. T. Chen, T. A. Abbott, E. Barrett-Connor, P. D. Miller, L. E. Wehren, M. L. Berger, Bone mineral density thresholds for pharmacological intervention to prevent fractures, Arch. Intern. Med. 164 (2004) 1108-1112.

[5] R. Barkmann, P. Laugier, U. Moser, S. Dencks, M. Klausner, F. Padilla, G. Haiat, C. C. Glüer, A device for in vivo measurements of quantitative ultrasound variables at the human proximal femur, IEEE Trans. Ultrason. Ferroelectr. Freq. Control 55 (2008) 1197-1204. Times Cited: 2 Article English Cited References Count: $20303 f$.

[6] R. Barkmann, S. Dencks, P. Laugier, F. Padilla, K. Brixen, J. Ryg, A. Seekamp, L. Mahlke, A. Bremer, M. Heller, C. C. Glüer, Femur ultrasound (FemUS) - first clinical results on hip fracture discrimination and estimation of femoral BMD, Osteoporos. Int. 21 (2010) 969-976.

[7] V. Bousson, A. Le Bras, F. Roqueplan, Y. Kang, D. Mitton, S. Kolta, C. Bergot, W. Skalli, E. Vicaut, W. Kalender, K. Engelke, J. D. Laredo, Volumetric quantitative computed tomography of the proximal femur: relationships linking geometric and densitometric variables to bone strength. Role for compact bone, Osteoporos. Int. 17 (2006) $855-64$.

[8] G. Holzer, G. von Skrbensky, L. A. Holzer, W. Pichl, Hip fractures and the contribution of cortical versus trabecular bone to femoral neck strength, J. Bone Miner. Res. 24 (2009) 468-474.

[9] J. Grondin, Q. Grimal, K. Engelke, P. Laugier, Potential of first arriving signal to assess cortical bone geometry at the hip with QUS: a model based study, Ultrasound Med. Biol. 36 (2010) 656-666. 
[10] Q. Grimal, D. Rohrbach, J. Grondin, R. Barkmann, C.-C. Glüer, K. Raum, P. Laugier, Modeling of femoral neck cortical bone for the numerical simulation of ultrasound propagation, Biomech. Model. Mechanobiol. (2013) submitted.

[11] Q. Grimal, J. Grondin, S. Guérard, R. Barkmann, K. Engelke, C.C. Glüer, P. Laugier, Quantitative ultrasound of cortical bone in the femoral neck predicts femur strength: results of a pilot study, J. Bone Miner. Res. 28 (2013).

[12] J. G. Minonzio, M. Talmant, P. Laugier, Guided wave phase velocity measurement using multi-emitter and multi-receiver arrays in the axial transmission configuration, J. Acoust. Soc. Am. 127 (2010) 2913-2919. Minonzio, Jean-Gabriel Talmant, Marilyne Laugier, Pascal.

[13] P. Nauleau, E. Cochard, J.-G. Minonzio, Q. Grimal, P. Laugier, C. Prada, Characterization of circumferential guided waves in a cortical bone-mimicking cylindrical phantom, J. Acoust. Soc. Am. 131 (2012) EL289-94.

[14] E. Bossy, SimSonic software suite for the simulation of ultrasound propagation, 2012. URL: http://www.simsonic.fr/.

[15] J. Virieux, SH-wave propagation in heterogeneous media: Velocitystress finite-difference method, Geophysics 49 (1984) 1933-1957.

[16] J. Virieux, P-SV wave propagation in heterogenous media: Velocitystress finite-difference method, Geophysics 51 (1986) 889-901.

[17] J. W. S. Rayleigh, The theory of sound, Dover, New-York, 1896.

[18] P. R. Stepanishen, The time-dependent force and radiation impedance on a piston in a rigid infinite planar baffle, J. Acoust. Soc. Am. 49 (1971) 841-849.

[19] D. Cassereau, G. Guyomar, Self and mutual time-dependent interaction forces between ultrasonic transducers - Application to the computation of radiation impedances, J. Acoust. Soc. Am. 93 (1993) 552-558.

[20] D. Cassereau, PASS - Phased Array Simulation Software, 1994-2013. URL: http://iadc.info.espci.fr/dc/pass/. 
[21] M. F. Hamilton, Comparison of three transient solutions for the axial pressure in a focused sound beam, J. Acoust. Soc. Am. 92 (1992) 527532.

[22] P. M. Morse, K. U. Ingard, Theoretical Acoustics, Mc-Graw-Hill, NewYork, 1968.

[23] A. Alastuey, M. Magro, P. Pujol, Physique et outils mathematiques : Methodes et exemples, EDP Sciences, 2008.

[24] F. Collino, C. Tsogka, Application of the PML absorbing layer model to the linear elastodynamic problem in anisotropic heterogeneous media, Geophysics 66 (2001) 294-307.

[25] J.-G. Minonzio, J. Foiret, M. Talmant, P. Laugier, Impact of attenuation on guided mode wavenumber measurement in axial transmission on bone mimicking plates, J. Acoust. Soc. Am. 130 (2011) 3574-3582.

[26] E. Bossy, Q. Grimal, Bone Quantitative Ultrasound, Springer, New York, 2010.

[27] C. Prada, J.-L. Thomas, M. Fink, The iterative time reversal process: Analysis of the convergence, The Journal of the Acoustical Society of America 97 (1995) 62-71.

[28] C. Prada, M. Fink, Separation of interfering acoustic scattered signals using the invariants of the time-reversal operator. application to Lamb waves characterization, J. Acoust. Soc. Am. 104 (1998) 801-807. Times Cited: 34 Article English Cited References Count: 20 108ff Part 1.

[29] B. Lombard, J. Piraux, Numerical treatment of two-dimensional interfaces for acoustic and elastic waves, J. Comput. Phys. 195 (2004) 90-116. OR 15 OR 15.

[30] B. Lombard, J. Piraux, C. Gélis, J. Virieux, Free and smooth boundaries in 2-D finite-difference schemes for transient elastic waves, Geophys. J. Int. 172 (2008) 252-261. Accepted and to be published in Geophys. J. Int. OR 15 OR 15.

[31] G. Luo, J. Kaufman, A. Chiabrera, B. Bianco, J. Kinney, D. Haupt, J. Ryaby, R. Siffert, Computational methods for ultrasonic bone assessment, Ultrasound Med. Biol. 25 (1999) 823-830. 
[32] E. Bossy, M. Talmant, P. Laugier, Effect of bone cortical thickness on velocity measurements using ultrasonic axial transmission: a $2 \mathrm{D}$ simulation study, J. Acoust. Soc. Am. 112 (2002) 297-307.

[33] A. Hosokawa, Simulation of ultrasound propagation through bovine cancellous bone using elastic and Biot's finite-difference time-domain methods, J. Acoust. Soc. Am. 118 (2005) 1782-1789.

[34] Y. Nagatani, H. Imaizumi, T. Fukuda, M. Matsukawa, Y. Watanabe, T. Otani, Applicability of finite-difference time-domain method to simulation of wave propagation in cancellous bone, Jpn. J. Appl. Phys. 1 45 (2006) 7186-7190. 